\title{
Quantum Monte Carlo calculation of the zero-temperature phase diagram of the two-component fermionic hard-core gas in two dimensions
}

\author{
N. D. Drummond \\ TCM Group, Cavendish Laboratory, University of Cambridge, J. J. Thomson Avenue, Cambridge CB3 OHE, United Kingdom \\ and Department of Physics, Lancaster University, Lancaster LA1 4YB, United Kingdom \\ N. R. Cooper and R. J. Needs \\ TCM Group, Cavendish Laboratory, University of Cambridge, J. J. Thomson Avenue, Cambridge CB3 OHE, United Kingdom
}

\author{
G. V. Shlyapnikov \\ Laboratoire de Physique Théorique et Modèles Statistiques, Université Paris Sud, CNRS, FR-91405 Orsay, France van der Waals-Zeeman \\ Institute, University of Amsterdam, Valckenierstraat 65/67, NL-1018 XE Amsterdam, The Netherlands
}

(Received 7 February 2011; revised manuscript received 14 April 2011; published 18 May 2011)

\begin{abstract}
Motivated by potential realizations in cold-atom or cold-molecule systems, we have performed quantum Monte Carlo simulations of two-component gases of fermions in two dimensions with hard-core interactions. We have determined the gross features of the zero-temperature phase diagram by investigating the relative stabilities of paramagnetic and ferromagnetic fluids and crystals. We have also examined the effect of including a pairwise, long-range $r^{-3}$ potential between the particles. Our most important conclusion is that there is no region of stability for a ferromagnetic fluid phase, even if the long-range interaction is present. We also present results for the pair-correlation function, static structure factor, and momentum density of two-dimensional hard-core fluids.
\end{abstract}

DOI: 10.1103/PhysRevB.83.195429

PACS number(s): 67.85.Lm, 02.70.Ss, 67.10.Db

\section{INTRODUCTION}

Ultracold gases of fermionic atoms and molecules have been the subject of a large number of experimental and theoretical studies ${ }^{1,2}$ in the last decade. The interest in these systems arises from the fact that the atoms or molecules obey quantum statistics rather than classical statistics and exhibit interesting and novel quantum phases at sufficiently low temperatures. Unlike electron systems, it is possible to manipulate the interaction between the atoms or molecules to some extent, e.g., by Feshbach resonances ${ }^{3}$ and/or by applying microwave fields. ${ }^{4-7}$ The ability to control the interactions can be used to gain insight into the physics of phase transitions and the nature of correlation effects in these quantum phases. Celebrated examples are the experimental studies of the Mott transition for bosons ${ }^{8}$ and of the superfluid pairing in the unitary (strong-coupling) Fermi gas. ${ }^{1,2}$ Finally, ultracold atomic gases may play an important role in future quantum computing devices.

Recently, interest has turned toward the use of ultracold atomic systems to investigate ferromagnetism in Fermi gases. Itinerant ferromagnetism in electron systems is poorly understood, and it is possible that insights might be gained by studying ferromagnetic fluid states in cold-atom systems. ${ }^{9}$ Experimental studies of strongly repulsive Fermi gases close to Feshbach resonances have found behavior consistent with the formation of (local) ferromagnetic correlations in the manner of Stoner ferromagnetism. ${ }^{10}$ However, these systems are limited by an intrinsic instability to molecule formation, which results in a short lifetime for the ferromagnetic state and may also complicate the interpretation of experimental features. ${ }^{11,12}$ It is therefore of interest to explore other forms of interaction that might give rise to ferromagnetism in the absence of nearby bound states.
An interesting class of experimental system in this regard is provided by gases of fermionic polar molecules (e.g., ${ }^{40} \mathrm{~K}^{87} \mathrm{Rb}$ or ${ }^{7} \mathrm{Li}^{40} \mathrm{~K}$ ). Polar molecules that are confined to two-dimensional (2D) layers and dressed by a circularly polarized microwave field experience a long-range potential that falls off as $r^{-3}$, where $r$ is the intermolecular distance. ${ }^{5-7}$ Furthermore, the dressed states have the feature that there is a very sharp increase in the potential energy at short distances, preventing close approach. Hence, such molecules can be modeled as a gas of fermionic hard-core particles with an additional long-range potential varying as $r^{-3}$. The hard-core potential prevents the formation of shallow bound states even for (weakly) attractive long-range interactions. It has been suggested that a single-component gas of such fermionic molecules will exhibit a topological superfluid $p$-wave pairing phase of direct relevance to possible topologically protected quantum computing devices. ${ }^{7,13}$

The $2 \mathrm{D}$ regime is reached by confining the motion of the molecules in one direction to zero-point oscillations. This requires the confinement frequency to be much larger than the Fermi energy. Generally, the confinement length ranges from 200-300 $\AA$ (for very tight confinement) to about $1 \mu \mathrm{m}$. The finite width leads to the appearance of a sort of effective core radius for hard-core interacting particles. This provides a natural estimate of the Fermi wavelength $\lambda_{F} \gtrsim 200-300 \AA$, for which a tightly confined system may be treated as strictly $2 \mathrm{D}$.

Here, we consider the case of a two-component gas of fermionic molecules moving in 2D. We have applied quantum Monte Carlo (QMC) methods to address the issue of whether or not the hard-core interactions cause this system to exhibit a region of itinerant ferromagnetism. In particular, we have used the variational and diffusion quantum Monte Carlo (VMC and DMC) techniques ${ }^{14,15}$ to establish the ground-state phase 


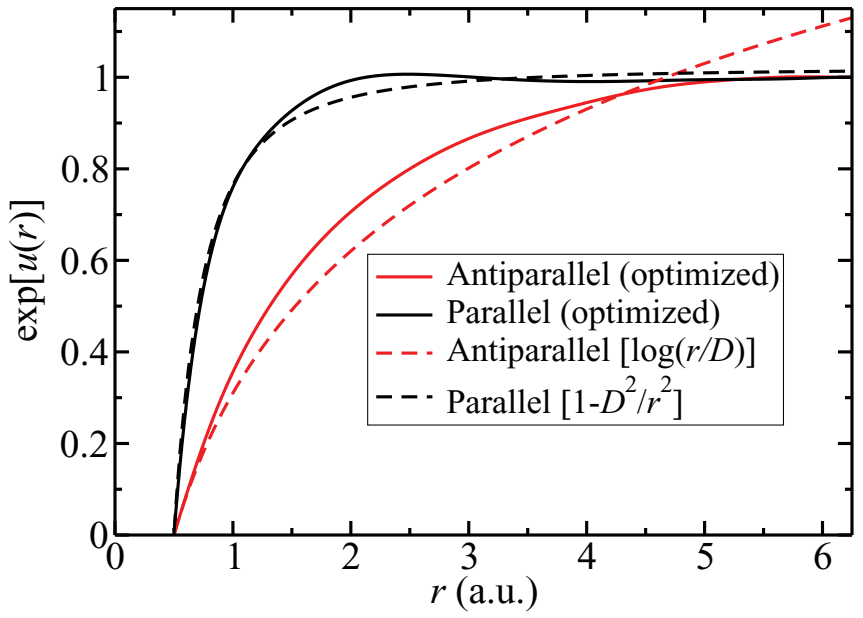

FIG. 1. (Color online) Two-body Jastrow factors $\exp [u(r)]$ for $N=50$ paramagnetic hard-core particles with $D=0.5$ a.u.

diagram of two-component gases of fermionic hard-core particles moving in 2D. QMC methods are widely acknowledged to be the most accurate first-principles techniques available for studying condensed matter. In the VMC method, Monte Carlo integration is used to evaluate expectation values with respect to an explicitly correlated many-body trial wave function. In the DMC method, the ground-state component of a trial wave function is projected out by a stochastic algorithm. Fermionic symmetry is maintained by the fixed-node approximation. ${ }^{16}$

Some of the earliest applications of QMC methods were to study the ground-state properties of bosonic hard-sphere fluids as a model for the behavior of ${ }^{4} \mathrm{He} \cdot{ }^{17-19}$ More recently, QMC methods have been used to study 3D fermionic systems in which there is a hard-sphere repulsion between opposite-spin particles. $^{20,21}$ To the best of our knowledge, however, QMC methods have never previously been used to study the phase diagram of 2D gases of fermionic hard-core particles

This paper is arranged as follows. In Sec. II, we describe the model Hamiltonian that we study. In Sec. III, we discuss the form of trial wave function that we use. In Sec. IV, we consider the issue of finite-size errors and explain how we extrapolate to the thermodynamic limit. In Sec. V, we present our results for the phase diagram of fermionic hard-core particles, and in Sec. VI, we present results for the pair-correlation function (PCF), static structure factor, and momentum density of hardcore fluids. In Sec. VII, we investigate whether the inclusion of weak, long-range interactions affects the phase diagram. Finally, we draw our conclusions in Sec. VIII. All our QMC calculations were performed using the CASINO code. ${ }^{22}$

\section{HARD-CORE MODEL}

\section{A. Hard-core Hamiltonian}

Suppose we have a system of interacting, circular hard-core particles moving in two dimensions. Throughout, we use units (a.u.) in which the Dirac constant, the mass of the particles, and the radius of the circle that contains one particle on average are unity. We assume the particles to be fermions with spin

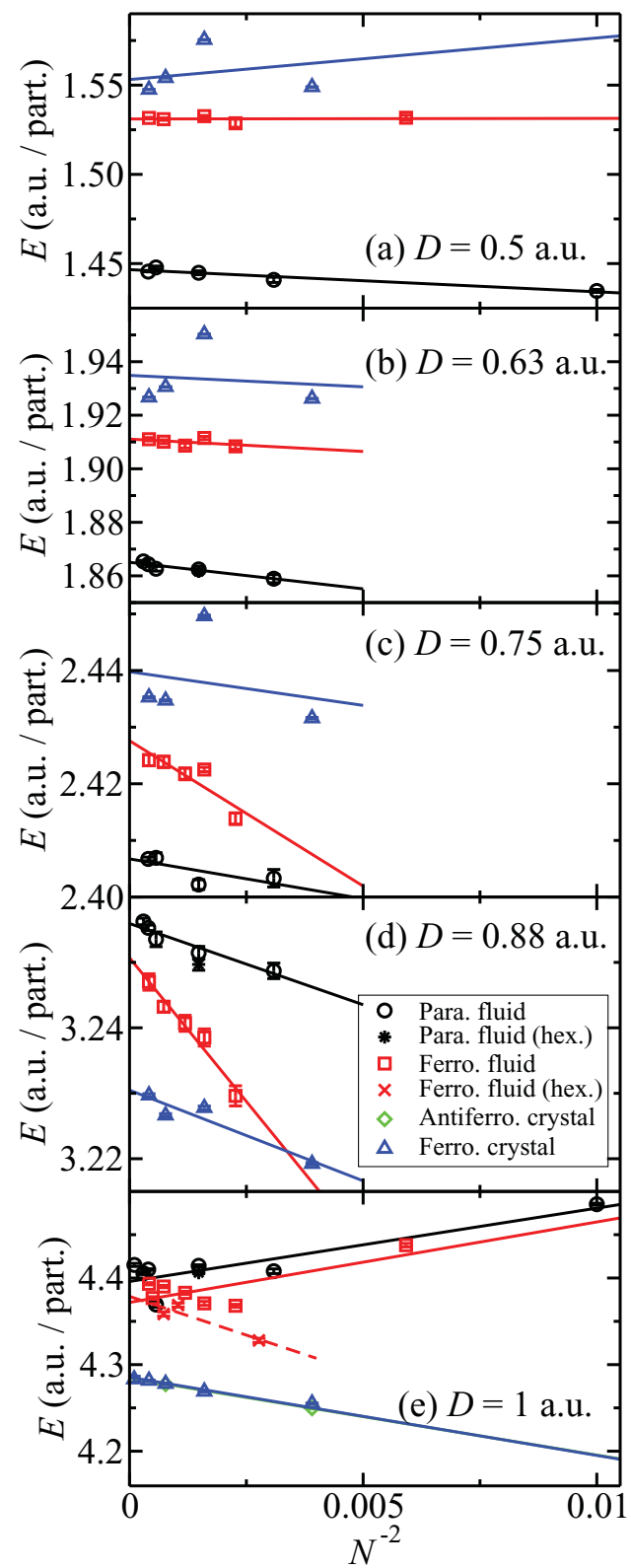

FIG. 2. (Color online) DMC energy $E$ against the reciprocal of the square of the system size $N^{-2}$ for (a) $D=0.5$ a.u., (b) $D=0.63$ a.u., (c) $D=0.75$ a.u., (d) $D=0.88$ a.u., and (e) $D=$ 1 a.u. The largest system size considered for ferromagnetic crystals is $N=49$ in each case, apart from $D=1$, where the largest size considered is $N=100$. The largest system size considered for the antiferromagnetic crystal is $N=36$. The largest system size considered for the paramagnetic fluid (square cell) is $N=50,58$, 50,58 , and 98 for $D=0.5,0.63,0.75,0.88$, and 1 , respectively. The largest system size considered for the ferromagnetic fluid is $N=49$ in each case.

$s=1 / 2$. Let $D$ be the diameter of the particles. In these units, the Hamiltonian is

$$
\hat{H}=\sum_{i}-\frac{1}{2} \nabla_{i}^{2}+\sum_{i>j} v_{H}\left(r_{i j}\right),
$$




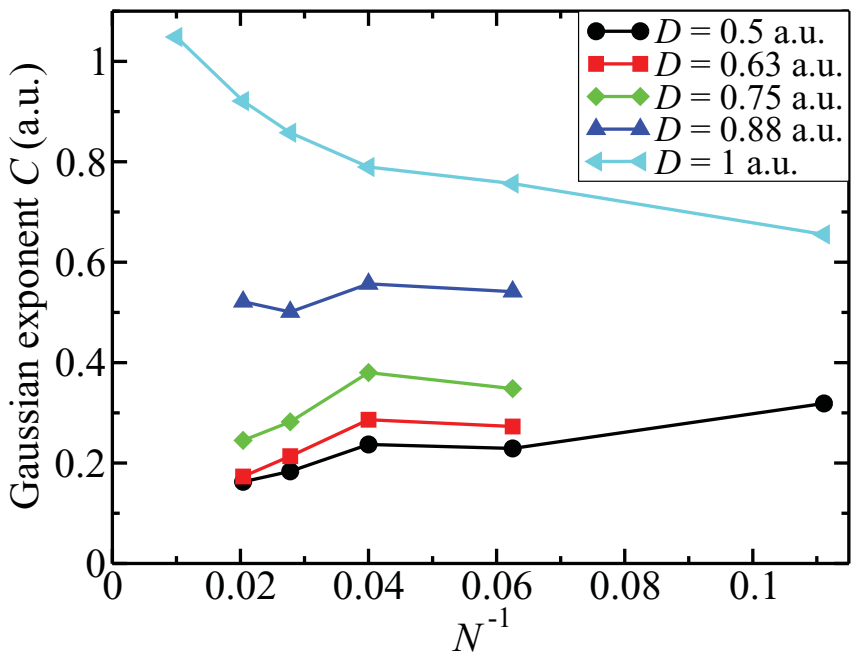

FIG. 3. (Color online) Optimal Gaussian exponent $C$ for the ferromagnetic crystal phase against the reciprocal of system size $N^{-1}$.

where

$$
v_{H}(r)= \begin{cases}0 & \text { if } r>D, \\ \infty & \text { otherwise. }\end{cases}
$$

When we refer to "high density," we mean that the value of $D$ is large (comparable with the radius of the circle that contains one particle on average). In our calculations, we used finite numbers $N$ of particles in cells subject to periodic boundary conditions. From these, we extrapolated to infinite system size, as discussed in Sec. IV. (The effects of an additional weak, long-range potential are discussed in Sec. VII.)

The potential energy is zero throughout the permitted region of configuration space. Hence, the expected potential energy is zero, and the system only has kinetic energy. Nevertheless, the hard-core interaction has a significant effect on the energy since it defines the boundary conditions on the solution of the Schrödinger equation. Hence, the system does not behave like

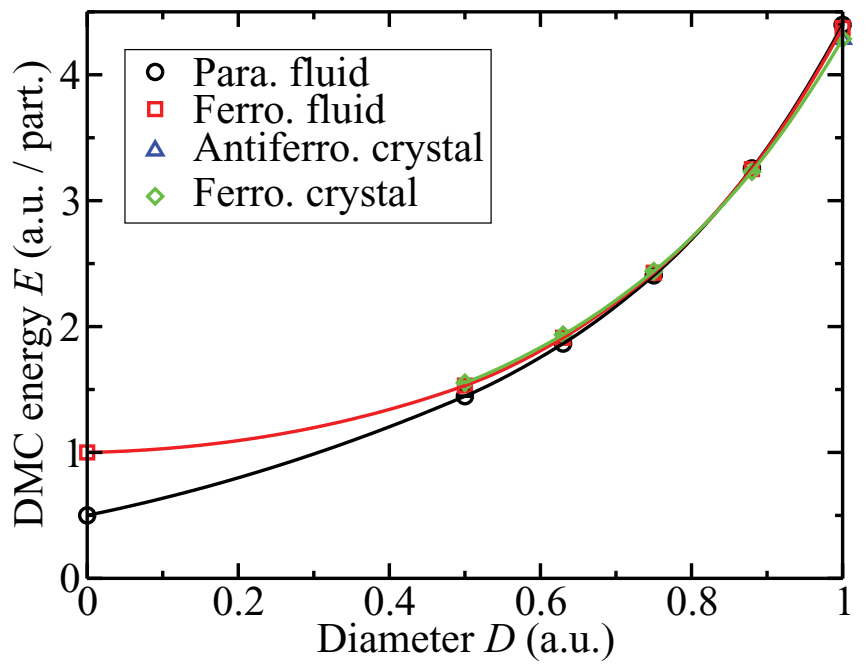

FIG. 4. (Color online) DMC energy $E$ against hard-core diameter $D$. Note that, when $D=0$, the energies of the paramagnetic and ferromagnetic fluids are 0.5 and 1 a.u. per particle, respectively.

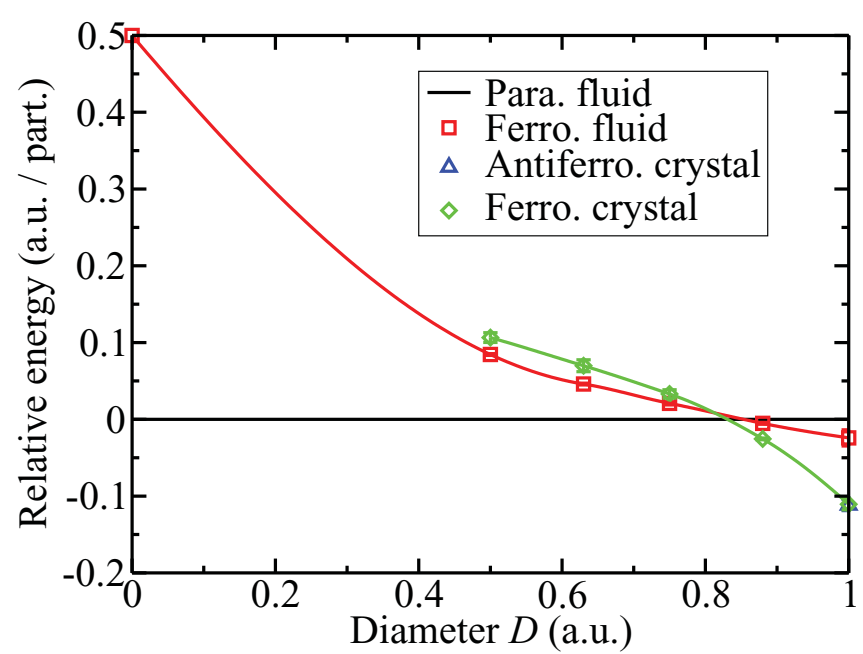

FIG. 5. (Color online) DMC energy of the different phases relative to the energy of the paramagnetic fluid.

a noninteracting Fermi gas: the magnetic behavior is nontrivial and the system must crystallize at a sufficiently high density.

Hard-core systems have a maximum density: the closepacking limit. In 2D, the triangular lattice has the highest packing fraction, with the maximum hard-core diameter being $D_{\text {max }}=\sqrt{2 \pi / \sqrt{3}}$.

\section{B. Qualitative features of the phase diagram}

For infinitesimal $D$, the system resembles a noninteracting Fermi gas. The momentum density is a unit step function. The paramagnetic fluid phase is favored because it has the lowest kinetic energy. The crystal is not even stable as an excited state for small $D$.

As $D$ is increased, the energy of the paramagnetic fluid rises more rapidly than that of the ferromagnetic fluid because wavefunction antisymmetry already keeps parallel-spin particles apart. The hard-core interactions have a greater effect on the distribution of antiparallel-spin particles, with the short-range PCF being forced to go to zero, as shown in Sec. VIA. The momentum density of a hard-core fluid with $D>0$ is not a unit step function, but it retains a discontinuity at the Fermi wave vector $k_{F}$. The crystal becomes stable as $D$ is increased, but with a higher energy than that of the fluid.

At large $D$, the energies of the ferromagnetic and (frustrated) antiferromagnetic crystals are very similar. At some value of $D$, the ferromagnetic fluid is lower in energy than the paramagnetic fluid, but at some other value of $D$, the crystal becomes more stable than either of the fluid phases. It is a nontrivial problem to determine which of these transitions occurs first. The crystal orbitals become delta functions in the close-packing limit.

\section{TRIAL WAVE FUNCTIONS}

\section{A. Slater-Jastrow-backflow wave functions}

We used trial wave functions of Slater-Jastrow-backflow form $\Psi=\exp (J) S^{\uparrow} S^{\downarrow}$. The Jastrow exponent $J$ included polynomial and plane-wave functions of the interparticle distances $^{23}$ together with a two-body term to impose the 

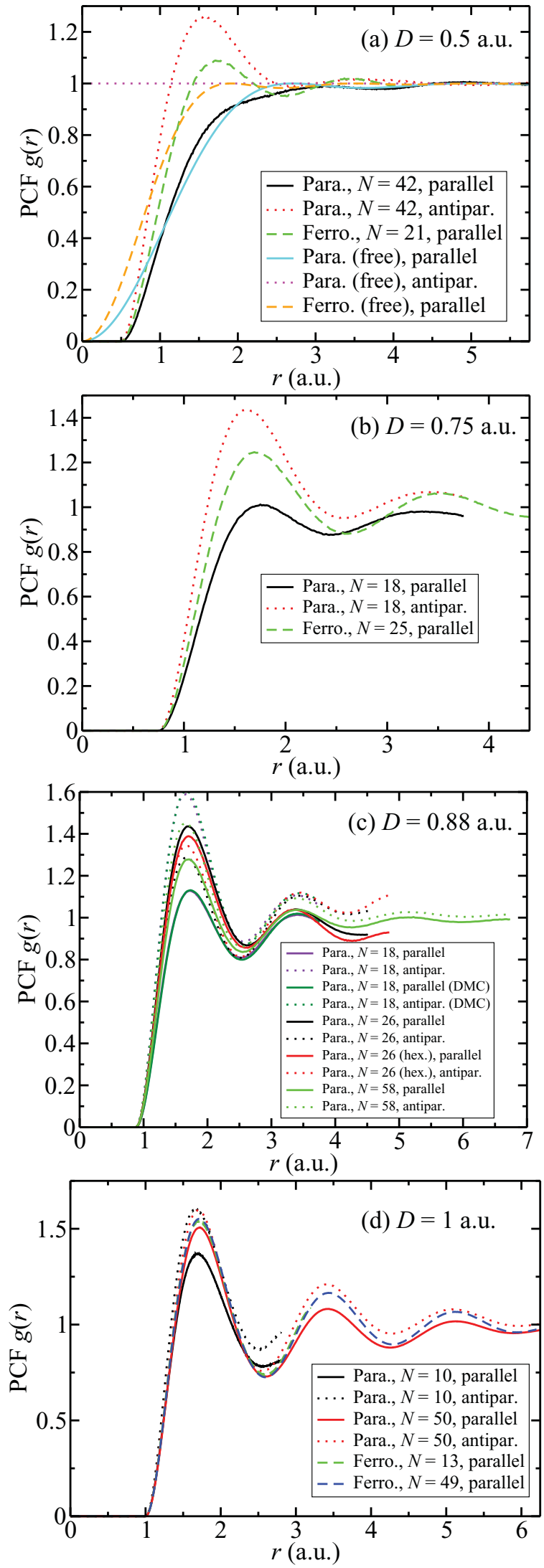

FIG. 6. (Color online) PCFs $g(r)$ of hard-core systems at (a) $D=0.5$ a.u., (b) $D=0.75$ a.u., (c) $D=0.88$ a.u., and (d) $D=$ 1 a.u. for different system sizes $N$. The results were all obtained with VMC except where indicated otherwise.

hard-core boundary conditions, as described in Sec. III B, and a three-body term. ${ }^{24}$ The backflow function consisted of a polynomial expansion in the interparticle distance. ${ }^{25}$ The terms in the Jastrow exponent and backflow function arising from parallel- and antiparallel-spin pairs of particles were allowed to differ.

For the fluid phases, we used plane-wave orbitals $\exp (i \mathbf{k} \cdot \mathbf{r})$ in the Slater determinants $S^{\uparrow}$ and $S^{\downarrow}$. The fluid phases suffer from momentum quantization effects (single-particle finitesize errors). To reduce these, we performed twist averaging in the canonical ensemble. ${ }^{26}$ For the crystal phases, we used Gaussian orbitals $\exp \left(-C\left|\mathbf{r}-\mathbf{R}_{p}\right|^{2}\right)$ centered on hexagonal lattice sites $\left\{\mathbf{R}_{p}\right\}$, where the exponent $C$ in the crystal orbitals was an optimizable parameter.

These calculations are similar to the QMC calculations that have been performed to establish the zero-temperature phase diagram of the three-dimensional homogeneous electron $\operatorname{gas}^{14,27,28}$ (HEG) and 2D HEG. ${ }^{29-31}$

\section{B. Hard-core two-body behavior}

\section{Antiparallel spins}

Let us rewrite the Schrödinger equation for two hardcore particles in terms of the center-of-mass and difference coordinates. We assume the center of mass is in its zero-energy ground state. The Schrödinger equation for the difference coordinate $\mathbf{r}$ is

$$
-\nabla^{2} \Psi=E \Psi
$$

For the boundary conditions, we assume that $\Psi=0$ at $r=D$ and $\Psi=0$ at $r=R$, where $R$ is very large. By increasing $R$, the ground-state eigenvalue $E$ can be made arbitrarily close to $0 . \Psi$ is circularly symmetric in the ground state for antiparallelspin particles, so

$$
-\frac{\partial^{2} \Psi}{\partial r^{2}}-\frac{1}{r} \frac{\partial \Psi}{\partial r} \approx 0
$$

with general solution $\Psi \approx A+B \log (r)$. This approximation is valid over any range of $r$ that is small compared with $R-$ $D$. However, we are interested in particle separations of the order $D$ and slightly larger. Applying the boundary condition $\Psi(D)=0$ gives

$$
\Psi(r) \propto \log (r / D),
$$

suggesting the antiparallel-spin two-body Jastrow factor in a hard-core gas should be $\log (r / D)$.

The two-body behavior of hard-core gases was studied in Ref. 32, but this work did not give the two-body Jastrow factor for the 2D gas and did not consider parallel-spin pairs.

\section{Parallel spins}

Suppose the two hard-core particles have parallel spins. In the lowest-energy state, the difference-coordinate wave function is of the form $\Psi=W(r) r \cos (\theta)$ and the energy eigenvalue is zero in the limit that the region over which the wave function is normalized is large. Hence, the Schrödinger equation for the radial part is

$$
-\frac{d^{2} W}{d r^{2}}-\frac{3}{r} \frac{d W}{d r} \approx 0 .
$$


The general solution over a range of $r$ values that is small compared with the region over which the wave function is normalized is $W(r) \approx A+B r^{-2}$. By applying the boundary condition $W(D)=0$, one obtains $W(r) \propto 1-D^{2} / r^{2}$. So, for small $r$,

$$
\Psi \propto\left(1-\frac{D^{2}}{r^{2}}\right) r \cos (\theta),
$$

suggesting the parallel-spin two-body Jastrow factor in a hardcore gas should be $1-D^{2} / r^{2}$.

\section{Hard-core Jastrow factor}

The wave function of a hard-core system must go linearly to zero as the separation of any pair of particles approaches $D$. To impose this behavior, the following term was included between all pairs of particles in the Jastrow exponent $J$ (in addition to the polynomial and plane-wave terms):

$$
u_{H}(r)=\left\{\begin{array}{lc}
-\infty & \text { if } r \leqslant D, \\
\log \left[\tanh \left(\frac{r / D-1}{\alpha\left(1-r / L_{\mathrm{WS}}\right)}\right)\right] & \text { if } D<r<L_{\mathrm{WS}}, \\
0 & \text { if } r \geqslant L_{\mathrm{WS}},
\end{array}\right.
$$

where $L_{\mathrm{WS}}$ is the radius of the circle inscribed in the WignerSeitz cell of the simulation cell. The parameter $\alpha$ was fixed at $1 ; u_{H}$ goes smoothly to zero at $L_{\mathrm{Ws}}$. The other terms in the Jastrow factor are analytic at $r=D$.

The $\exp \left(u_{H}\right) \approx \tanh [(r-D) / D]$ is not of the form $\log (r / D)$ or $1-D^{2} / r^{2}$ suggested by the analytic results for opposite-spin or same-spin hard-core particles, respectively, but the short-range behavior is correct [linear in $(r-D)$ in the vicinity of $r=D$ ]. When optimized, the polynomial and plane-wave terms in the Jastrow exponent describe all two-body correlations. Optimized two-body Jastrow factors are plotted in Fig. 1, confirming that the simple theory of two-body correlations described in Sec. III B is approximately valid.

\section{Free-particle limit}

It is interesting to consider the approach to the free-particle limit as the diameter $D$ tends to zero. In a three-dimensional hard-core gas, similar arguments to those given in Secs. III B 1 and III B 2 show that the two-body Jastrow factors are approximately $1-D / r$ and $1-D^{3} / r^{3}$ for antiparallel and parallel spins, respectively, both of which tend to unity as $D \rightarrow 0$. Hence, a Slater-Jastrow wave function for a three-dimensional hard-core fluid reduces to a Slater determinant of plane-wave orbitals in the limit of zero particle diameter. In the 2D hard-core gas, the two-body Jastrow factors are approximately $\log (r / D)$ and $1-D^{2} / r^{2}$ for antiparallel and parallel spins, respectively. The antiparallel-spin two-body Jastrow factor is the marginal case in which two-body correlations become negligible over any given length scale as $D$ is made small so that free-particle behavior is recovered in the $D \rightarrow 0$ limit. In one dimension, however, the infinite contact potential that remains when the $D \rightarrow 0$ limit is taken prevents the hard-core gas from exhibiting free-particle behavior, unless the system is fully ferromagnetic.

\section{E. Behavior of the local energy as hard-core particles collide}

Suppose two antiparallel-spin hard-core particles 1 and 2 approach each other, i.e., their separation approaches $D$. Their contribution to the Jastrow exponent is $J_{12}=\log \left(r_{12}-D\right)$. Let us write the trial wave function as $\Psi=\exp \left(J_{12}\right) S$, where $S$ is well behaved as the particles approach. Suppose that all coordinates in the system are frozen, apart from the separation of particles 1 and 2 . The contribution to the local energy arising from this coordinate is

$$
\begin{aligned}
E_{L 12} & =-\frac{\nabla_{12}^{2} \Psi}{\Psi} \\
& =\left|\nabla_{12} J_{12}\right|^{2}+\nabla_{12}^{2} J_{12}+2 \nabla_{12} J_{12} \cdot \frac{\nabla_{12} S}{S}+\frac{\nabla_{12}^{2} S}{S} \\
& =\frac{1}{r_{12}\left(r_{12}-D\right)}\left(1+2 \mathbf{r}_{12} \cdot \frac{\nabla_{12} S}{S}\right)+\frac{\nabla_{12}^{2} S}{S}
\end{aligned}
$$

The local energy diverges when hard-core particles approach one another, and the sign of the divergence depends on the positions of all the particles. Hence, one can not remove the divergence using a two-body Jastrow factor. By contrast, when two charged point particles approach one another, the divergence in the local energy can be removed by imposing the Kato cusp conditions via the two-body Jastrow factor. ${ }^{33}$

The divergence is, in principle, no worse than that which occurs at any other node. However, there are many extra nodes introduced by the hard-core potentials on which the local kinetic energy diverges. These nodes can cause DMC population explosions, ${ }^{34}$ especially if three-body terms are omitted from the Jastrow factor.

\section{F. Need for a three-body Jastrow term}

Physically, it is obvious that, at high densities, multibody correlation effects will be important: motion is only possible if the particles move collectively. In fact, as shown in Table I, three-body Jastrow terms lower the VMC energy more than backflow. This differs from the behavior found in the HEG. ${ }^{35}$ We have therefore used three-body terms in all our calculations. If the range of the three-body terms is restricted, then the wave function becomes significantly poorer, as can be seen in Table I. Unfortunately, the need to include long-range three-body terms in the Jastrow factor makes the calculations expensive.

\section{G. Spin dependence in paramagnetic phases}

We used separate two-body Jastrow and two-body backflow terms for parallel- and antiparallel-spin pairs of particles in all our calculations. We considered two possible spin dependences for the three-body terms: either (A) using the same three-body term for all triples of particles or (B) using separate threebody terms for (i) triples involving three particles of the same spin and (ii) triples involving two particles of one spin and one particle of the opposite spin. VMC results obtained using these two different possibilities are shown in Table II. It is clear that allowing different three-body terms for different spin configurations lowers the variational energy significantly (although not by nearly as much as lifting the restriction on the 
TABLE I. Non-twist-averaged VMC and DMC results with different wave functions ("WF") for a paramagnetic fluid of $N=26$ hard-core particles of diameter $D=1$ a.u. The "SR BF" column specifies whether or not the backflow function was restricted to be short ranged (cutoff length 2.5 a.u.), while the "SR 3BJ" column specifies whether or not the three-body Jastrow terms were restricted to be short ranged (cutoff length 2.5 a.u.). Where the cutoff lengths were not restricted, they approached the radius of the circle inscribed in the Wigner-Seitz cell of the simulation cell. "T" and "F" denote true and false, while "Var." is the variance of the local energy.

\begin{tabular}{lccclc}
\hline \hline Method & WF & SR BF & SR 3BJ & $E$ (a.u./part.) & Var. (a.u.) \\
\hline VMC & SJ & & & $4.8242(4)$ & 286 \\
VMC & SJB & F & & $4.7172(9)$ & 242 \\
VMC & SJB3 & T & T & $4.6360(2)$ & 160 \\
VMC & SJB3 & F & T & $4.6272(1)$ & 158 \\
VMC & SJ3 & & F & $4.58672(8)$ & 89 \\
VMC & SJB3 & T & F & $4.5201(2)$ & 70 \\
VMC & SJB3 & F & F & $4.5072(6)$ & 65 \\
DMC & SJ & & & $4.4157(6)$ & \\
DMC & SJB & F & & $4.4058(7)$ & \\
DMC & SJB3 & F & F & $4.3930(8)$ & \\
\hline \hline
\end{tabular}

range of the three-body terms) and hence we have used spin dependence $\mathrm{B}$ in all our production calculations.

\section{H. Relative accuracy of wave functions for different phases}

The variance of the energy is zero if the trial wave function is an eigenfunction of the Hamiltonian. As shown in Table III, the variance per particle is significantly lower for the ferromagnetic fluid than for the paramagnetic fluid, indicating that the trial wave function is more accurate for the former than the latter. The variance per particle is similar for ferromagnetic fluids and crystals near the transition density. Hence, if anything, our results are biased in favor of ferromagnetic phases.

\section{Nature of phase transition}

We have looked for a first-order phase transition by comparing fixed-node DMC energies with fluid and crystal orbitals, relying on the fixed-node approximation to impose the symmetry of the phase on the wave function. However, it

TABLE II. Non-twist-averaged VMC results with different spin dependences for a paramagnetic fluid of $N=26$ hard-core particles of diameter $D=1$ a.u. The "SR BF \& 3BJ" column specifies whether or not the backflow function and three-body Jastrow terms were restricted to be short ranged (cutoff length 2.5 a.u.). Where the cutoff lengths were not restricted, they approached the radius of the circle inscribed in the Wigner-Seitz cell of the simulation cell. " $T$ " and " $F$ " denote true and false, while "Var." is the variance of the local energy.

\begin{tabular}{lclc}
\hline \hline Spin dependence & SR BF \& 3BJ & $E$ (a.u./part.) & Var. (a.u.) \\
\hline A & T & $4.640(1)$ & 165 \\
B & T & $4.6360(2)$ & 160 \\
A & F & $4.534(1)$ & 76 \\
B & F & $4.5072(6)$ & 65 \\
\hline \hline
\end{tabular}

TABLE III. Non-twist-averaged VMC results for paramagnetic fluids with $N=26$ hard-core particles and ferromagnetic fluids with $N=25$ hard-core particles.

\begin{tabular}{lll}
\hline \hline$D$ (a.u.) & Phase & Var. per part. (a.u.) \\
\hline 1 & Paramagnetic fluid & 2.49 \\
1 & Ferromagnetic fluid & 1.76 \\
1 & Ferromagnetic crystal & 0.736 \\
0.88 & Paramagnetic fluid & 1.34 \\
0.88 & Ferromagnetic fluid & 0.616 \\
0.88 & Ferromagnetic crystal & 0.494 \\
0.63 & Paramagnetic fluid & 0.385 \\
0.63 & Ferromagnetic fluid & 0.147 \\
0.63 & Ferromagnetic crystal & 0.228 \\
\hline \hline
\end{tabular}

is possible that there could actually be a continuous transition from fluid to crystal behavior. The fact that the "fluid" wave function tries to become crystal-like at high density and the "crystal" wave function tries to become fluidlike at low density (see Sec. VI) supports this view. Nevertheless, even if this is the case, our calculations determine the region in which crystallization is expected to take place, and demonstrate that a ferromagnetic fluid phase is unlikely to occur.

\section{FINITE-SIZE EFFECTS}

According to the simple theory given in Sec. III B 2, the long-range parallel-spin two-body Jastrow exponent is approximately given by

$$
u_{\alpha \alpha}(r)=\log \left(1-D^{2} / r^{2}\right) \approx-D^{2} / r^{2}+O\left(r^{-4}\right) .
$$

The 2D Fourier transform of the leading term does not have a power-law behavior. In fact, numerical tests show that $\lim _{\alpha \rightarrow 0} \int r^{-2} \exp (i \mathbf{k} \cdot \mathbf{r}-\alpha r) d \mathbf{r}$ diverges logarithmically at small $k$. So, for small $k, u_{\alpha \alpha}(k)$ may be written as $-c D^{2} \log (k)$, where $c$ is a positive constant.

The Chiesa-Holzmann-Martin-Ceperley ${ }^{36}$ approximation to the long-range two-body finite-size correction to the kinetic energy in $2 \mathrm{D}$ is

$$
\begin{aligned}
\Delta T & =-\sum_{\alpha} \frac{N_{\alpha}}{4(2 \pi)^{2}} \int_{0}^{Q} 2 \pi k k^{2} u_{\alpha \alpha}(k) d k \\
& =-\frac{N}{8 \pi} \int_{0}^{Q} k^{3} u_{\alpha \alpha}(k) d k,
\end{aligned}
$$

where $Q=2 \sqrt{\pi / A}$ is the radius of the circle in $\mathbf{k}$ space with area $(2 \pi)^{2} / A$, and $A=\pi N$ is the area of the simulation cell. Inserting $u_{\alpha \alpha}(k)=-c D^{2} \log (k)$ gives

$$
\begin{aligned}
\Delta T & \approx \frac{\pi N c D^{2}}{2 A^{2}}\left[\log (2 \sqrt{\pi / A})-4 \pi^{2} / A^{2}\right] \\
& =O\left[D^{2} N^{-1} \log (N)\right] .
\end{aligned}
$$

Hence, ignoring the logarithmic factor, the leading-order correction to the energy per particle due to the neglect of longrange two-body correlations falls off as $O\left(N^{-2}\right)$. This is much more rapid than the analogous result for the $2 \mathrm{D} \mathrm{HEG}{ }^{37}$ The leading-order correction is positive. Unsurprisingly, however, two-body finite-size errors get rapidly more severe as $D$ increases. 


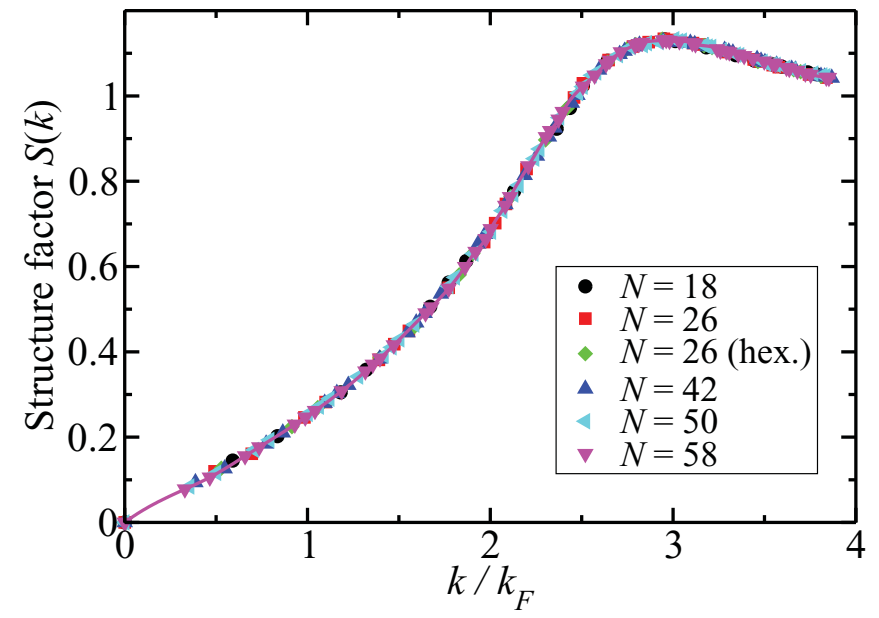

FIG. 7. (Color online) VMC structure factor of the paramagnetic fluid at $D=0.63$ a.u. for a range of system sizes $N$.

We have carried out numerical tests that show that the bias due to twist averaging in the canonical ensemble in two dimensions falls off as $O\left(N^{-3 / 2}\right)$, but with a small prefactor. Residual single-particle errors in the canonicalensemble twist-averaged fluid energies are small compared with the effect of twist averaging, and the difference between the twist-averaged and non-twist-averaged energies is only about 0.02 a.u. per particle at $D=1$ a.u. for $N=10$ and 26 . Hence, the $O\left(N^{-2}\right)$ behavior due to the neglect of long-range two-body correlations dominates the systematic error for all the hard-core diameters $D$ that we have studied, as can be seen in Fig. 2.

Several data points in Fig. 2 are outliers. This nonsystematic behavior appears to be a genuine finite-size effect. The finite-size "noise" in the crystal energies is more severe at low densities, while the finite-size noise in the fluid energies is more severe at very high densities. The theory of two-body finite-size effects developed above breaks down for fluids at high density and crystals at low density: the sign of the bias is wrong. These cases are pathological in various respects. At very high densities, the fluid energies obtained with the same number of particles in different-shaped cells disagree, although they extrapolate to the same value in the limit of infinite system size. In fact, at very high density, it is geometrically impossible to fit finite fluids in some cell shapes. As can be seen in Fig. 3, the Gaussian exponent $C$ of the crystal is not well behaved for $D \leqslant 0.75$, suggesting that the crystal is becoming unstable. As shown in Fig. 15, the momentum density is developing an edge, leading to substantial single-particle finite-size errors.

As shown in Table I, restricting the range of the three-body term raises the variational energy significantly. Three-body correlations are therefore long ranged. So, there must be threebody finite-size errors in the fluid and crystal energies obtained in finite simulation cells. We assume that they are a "random" error about the systematic finite-size bias due to two-body finite-size effects. We have therefore obtained several $E(N)$ data points for each phase at each density in order to average out the "noise" when extrapolating to infinite system size.

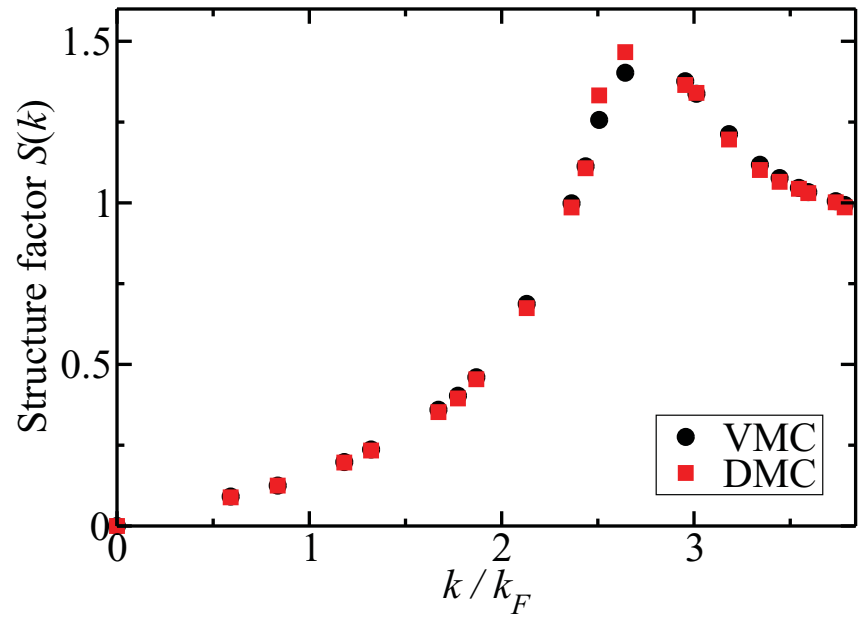

FIG. 8. (Color online) VMC and DMC structure factors of the paramagnetic fluid at $D=0.88$ a.u. with $N=18$ particles.

\section{PHASE DIAGRAM}

The energies of the different phases are compared in Fig. 4 and are plotted relative to the energy of the paramagnetic fluid phase in Fig. 5. The continuous curves shown are Akima spline interpolations between the DMC energies. (Akima spline interpolation is stable to the presence of outliers in the data. ${ }^{38}$ ) It can be seen that crystallization takes place when $D=$ 0.83 a.u., leaving no region of stability for a ferromagnetic fluid. (Recall that our calculations are, if anything, biased in favor of the ferromagnetic fluid.) Thus, our calculations rule out the possibility of an itinerant ferromagnetic fluid phase in a 2D gas of particles with only hard-core interactions. For those values of $D$ for which the crystal has the lowest energy, the energy difference between the antiferromagnetic ${ }^{39}$ and ferromagnetic states is insignificant, showing that exchange interactions in the crystalline phase are negligible.

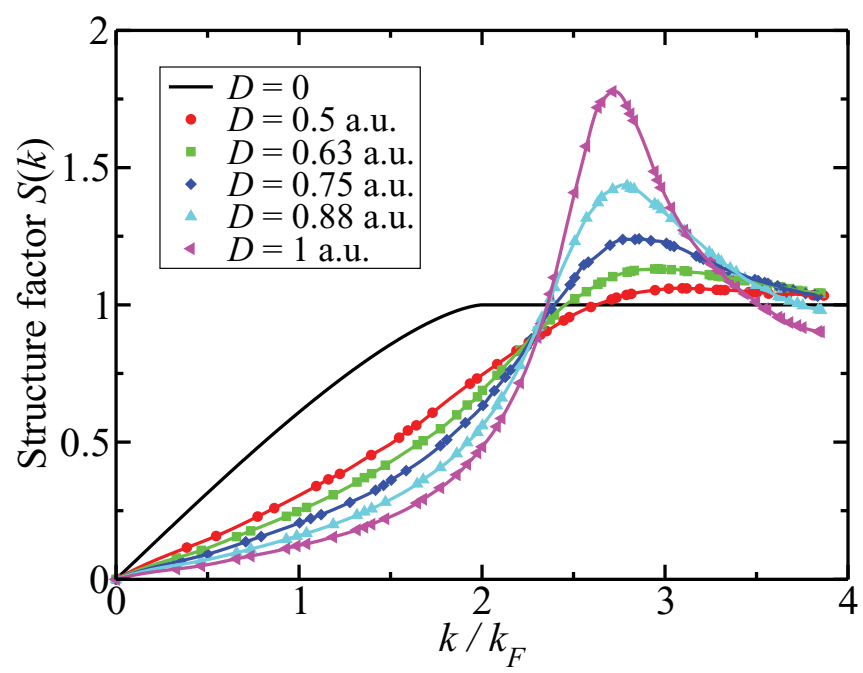

FIG. 9. (Color online) VMC structure factors of paramagnetic fluids. For $D=0$, the analytic result for a paramagnetic free-particle gas is given. 


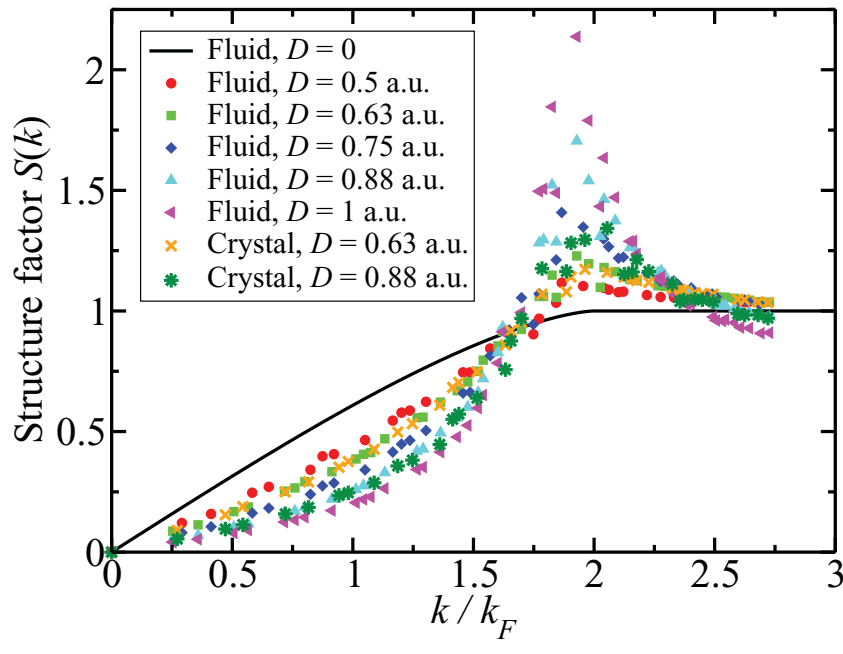

FIG. 10. (Color online) VMC structure factors of ferromagnetic fluids and crystals. The $D=0$ result is the analytic structure factor for a ferromagnetic free-particle gas.

\section{OTHER PROPERTIES OF HARD-CORE GASES}

\section{A. Pair-correlation function}

We have calculated the PCFs of the fluid phases of the hard-core gas, and our results are shown in Fig. 6. The PCFs, which were obtained without twist averaging, are not well converged with respect to system size at high density: the finite-size errors are oscillatory. Nevertheless, we can make some qualitative comments about the physics revealed by the PCF. It can be seen that there is excellent agreement between the VMC and DMC results where the latter are available, confirming that our trial wave functions are of high quality.

The distance between the peaks of the PCF obtained in square cells at $D=1$ a.u. is $\sqrt{\pi}$, which arises from the squarecell geometry. The difference between the PCFs in hexagonal and square cells is still significant at $D=0.88$ a.u. and $N=$ 26. The antiparallel-spin PCF evolves slowly toward $g(r)=$ 1 (the free-particle result) as $D$ is reduced. The size of the exchange-correlation hole and the ripples in the parallel-spin PCF are determined by the Fermi wave vector $k_{F}$ when $D$ is small.

\section{B. Static structure factor}

The static structure factor of a paramagnetic fluid at $D=$ 0.63 a.u. for various values of $N$ is plotted in Fig. 7. The structure factor is well converged with respect to $N$. VMC and DMC structure factors are compared in Fig. 8. The VMC and DMC results are in close agreement everywhere except in the immediate vicinity of the peak.

VMC-calculated structure factors for paramagnetic and ferromagnetic hard-core gases are shown in Figs. 9 and 10, respectively. Finite-size "noise" is much worse for ferromagnetic phases. The fluid and crystal structure factors are similar, especially at low densities.

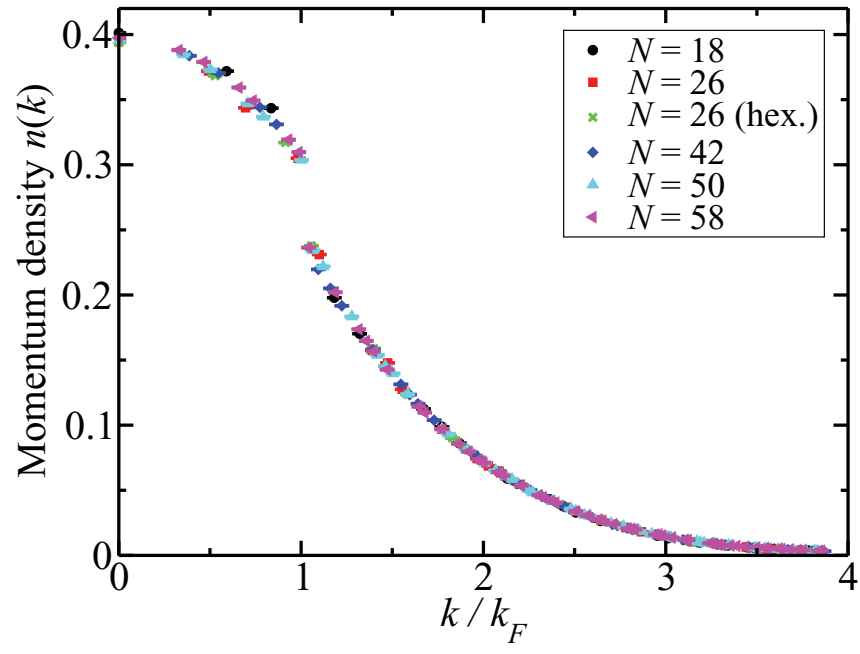

FIG. 11. (Color online) VMC momentum density of the paramagnetic fluid at $D=0.88$ a.u. at different system sizes $N$.

\section{Momentum density}

\section{Results for fluids}

The momentum density shown in Fig. 11 is reasonably well converged with respect to $N$. VMC results for the momentum densities of paramagnetic and ferromagnetic hardcore systems are shown in Figs. 12 and 13, respectively.

\section{Renormalization factors}

The renormalization factor, the discontinuity $Z$ in the momentum density at the Fermi edge, is plotted against hard-core diameter in Fig. 14. The finite-size errors in $Z$ are oscillatory, so we have averaged over the results obtained for different system sizes $N$.

\section{Results for crystals}

The momentum densities of ferromagnetic hard-core crystals are shown in Fig. 15. This figure strongly confirms the conclusion that the crystal is unstable at $D=0.63$ a.u.: the

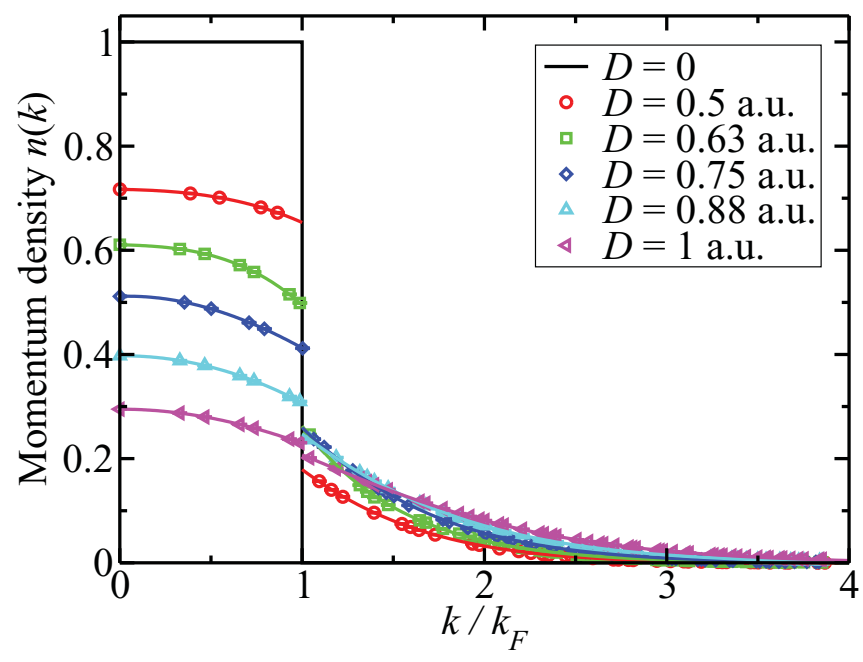

FIG. 12. (Color online) VMC momentum densities of paramagnetic fluids at different hard-core diameters $D$. 


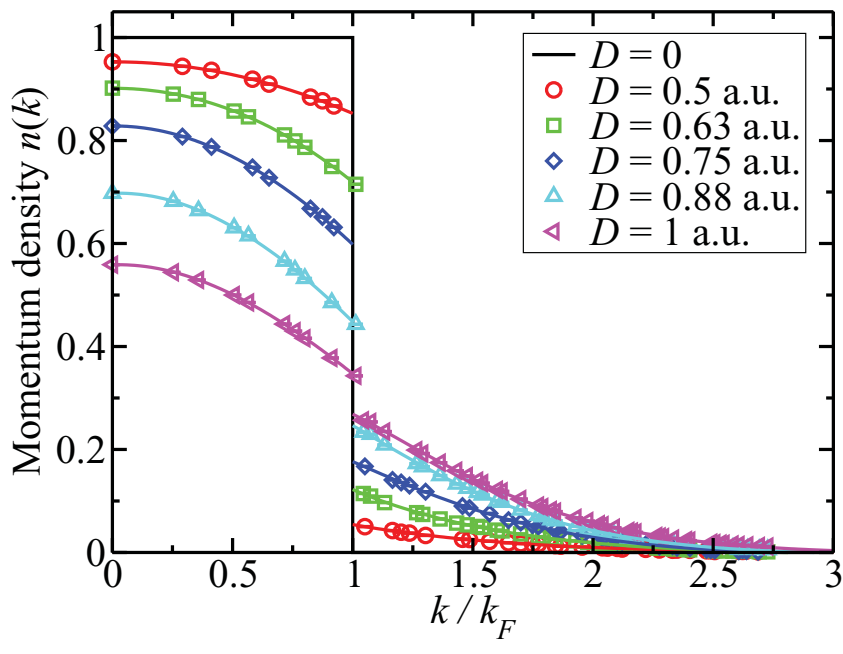

FIG. 13. (Color online) VMC momentum densities of ferromagnetic fluids at different hard-core diameters $D$.

"crystal" momentum density develops a near discontinuity at the Fermi wave vector.

\section{WEAK, LONG-RANGE INTERACTIONS}

\section{A. Form of the interaction}

Our results show that, close to the transition from fluid to crystal, the energies of the paramagnetic and ferromagnetic fluids are very finely balanced, albeit with the paramagnetic fluid always lying lower in energy. It is interesting to ask whether a small change in the two-body potential might alter the relative stabilities of these two fluid phases and allow a region of stable ferromagnetic fluid.

As explained in Sec. I, it can be arranged that fermionic molecules confined to a plane experience an additional longrange, pairwise interaction varying as $\Lambda r^{-3}$, where $r$ is the interparticle separation and $\Lambda$ is a constant. ${ }^{5,7}$ In a finite, periodic cell, the two-body interaction between one particle

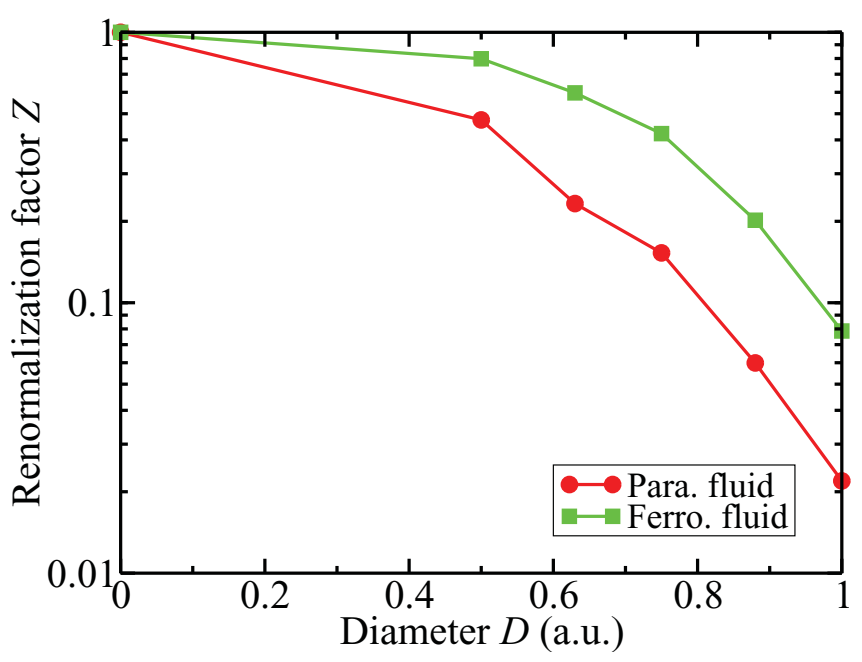

FIG. 14. (Color online) Renormalization factor $Z$ against $D$, from fits to the momentum density.

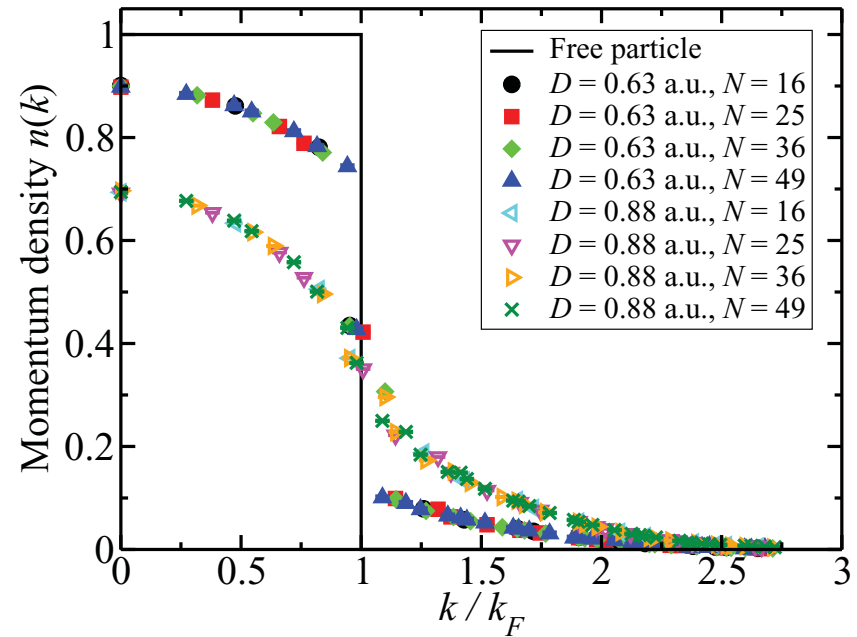

FIG. 15. (Color online) VMC momentum densities of ferromagnetic crystals.

and all the images of another particle that is at a distance $\mathbf{r}$ from the first is

$$
\begin{aligned}
v_{A}(\mathbf{r}) & =\sum_{\mathbf{R}} \frac{\Lambda}{|\mathbf{r}+\mathbf{R}|^{3}} \\
& \approx \sum_{\mathbf{R} \in S} \frac{\Lambda}{|\tilde{\mathbf{r}}+\mathbf{R}|^{3}}+\frac{\Lambda}{A} \int_{R>R_{S}} \frac{d \mathbf{R}}{|\tilde{\mathbf{r}}+\mathbf{R}|^{3}} \\
& \approx \sum_{\mathbf{R} \in S} \frac{\Lambda}{|\tilde{\mathbf{r}}+\mathbf{R}|^{3}}+\frac{2 \pi \Lambda}{A R_{S}}\left(1+\frac{3 \tilde{r}^{2}}{4 R_{S}^{2}}\right)+O\left(R_{S}^{-5}\right),
\end{aligned}
$$

where $S$ is a circular region of radius $R_{S}$ centered on the origin, and $\tilde{\mathbf{r}}$ is the minimum image of $\mathbf{r}$. By making $R_{S}$ sufficiently large, the approximation to the infinite sum can be made arbitrarily good.

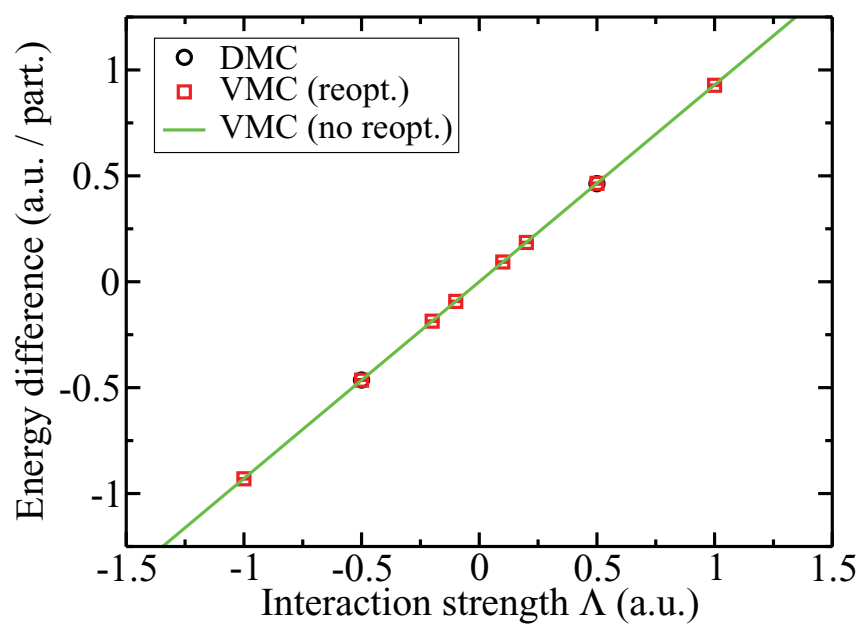

FIG. 16. (Color online) Change in energy resulting from the inclusion of a $\Lambda r^{-3}$ interaction for a paramagnetic fluid of $N=18$ hard-core particles of diameter $D=0.88$ a.u. 
The "Madelung" term (the interaction of each particle with its own images) is

$$
\begin{aligned}
v_{M} & =\sum_{\mathbf{R} \neq \mathbf{0}} \frac{\Lambda}{|\mathbf{R}|^{3}} \\
& \approx \sum_{\mathbf{R} \in S-\{\mathbf{0}\}} \frac{\Lambda}{|\mathbf{R}|^{3}}+\frac{\Lambda}{A} \int_{R>R_{S}} \frac{d \mathbf{R}}{|\mathbf{R}|^{3}} \\
& \approx \sum_{\mathbf{R} \in S-\{\mathbf{0}\}} \frac{\Lambda}{|\mathbf{R}|^{3}}+\frac{2 \pi \Lambda}{A R_{S}} .
\end{aligned}
$$

The Hamiltonian for the finite, periodic cell is therefore

$$
\hat{H}=-\frac{1}{2} \sum_{i} \nabla_{i}^{2}+\sum_{i>j}\left[v_{H}\left(\left|\tilde{\mathbf{r}}_{i j}\right|\right)+v_{A}\left(\tilde{\mathbf{r}}_{i j}\right)\right]+\frac{N v_{M}}{2} \text {. }
$$

\section{B. Convergence of the real-space sum}

We have chosen $R_{S}$ such that 119 stars of lattice vectors are summed over explicitly in square cells (for fluid phases) and 86 stars are summed over in hexagonal cells (for crystal phases). The error in the Madelung constant is about $2 \times$ $10^{-7}$ a.u. per particle for $N=18$ particles (and smaller at larger $N$ ). The error in the additional interaction per particle due to the finite number of stars of $\mathbf{R}$ vectors should therefore be much smaller than $10^{-6}$ a.u. The error made in truncating the real-space sum is therefore negligibly small compared with the statistical error bars on our QMC energies.

\section{Validity of perturbation theory}

To a very good approximation, we can describe the effect of the $\Lambda r^{-3}$ interaction using perturbation theory. The change in the energy resulting from the additional interaction is approximately given by the expectation of the interaction operator with respect to the previously optimized wave function for the unperturbed system, which can be evaluated using VMC. For $-1<\Lambda<1$, this approximation reproduces the full DMC energy difference to within about 0.002 a.u. at $D=0.88$ a.u., as shown in Fig. 16 .

\section{Finite-size errors}

As with total energies, finite-size errors in the expectation value of the extra interaction are larger and quasirandom for the unstable phases. We extrapolate to infinite system size in each case, as shown in Fig. 17.

\section{E. Effect of weak interactions on the phase diagram}

At $D=0.88$ a.u., combining the VMC results for $\left\langle\sum_{i>j} r_{i j}^{-3}\right\rangle$ with the DMC energies in the absence of the additional interaction, we find that

$$
\begin{aligned}
& E_{\mathrm{PF}}=3.2559+0.9296 \Lambda, \\
& E_{\mathrm{FF}}=3.251+0.928 \Lambda, \\
& E_{\mathrm{FC}}=3.2305+0.9244 \Lambda,
\end{aligned}
$$

where $E_{\mathrm{PF}}, E_{\mathrm{FF}}$, and $E_{\mathrm{FC}}$ are the energies in a.u. per particle for the paramagnetic fluid, ferromagnetic fluid, and ferromagnetic

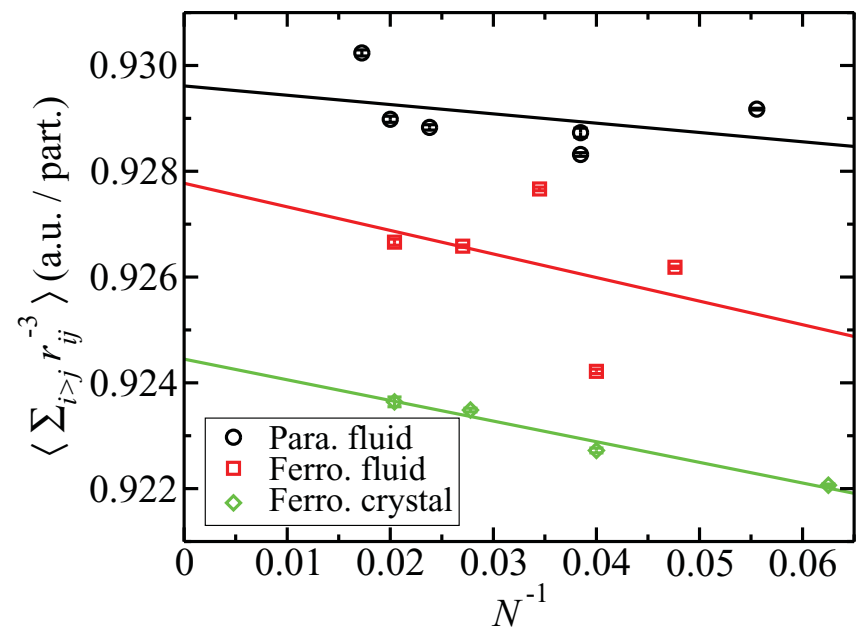

FIG. 17. (Color online) VMC-evaluated expectation value of $\sum_{i>j} r_{i j}^{-3}$ with respect to the nonreoptimized VMC wave function.

crystals, respectively, as a function of $\Lambda$. The resulting offset in the energy relative to the energy of the paramagnetic fluid phase at a density close to the crystallization density is shown in Fig. 18. The changes in the energies of the three phases due to the inclusion of the $\Lambda r^{-3}$ tail are very similar. As one would expect, a repulsive potential $(\Lambda>0)$ favors phases where the particles are kept apart (i.e., ferromagnetic over paramagnetic phases, and crystals over fluids), whereas an attractive interaction $(\Lambda<0)$ favors a paramagnetic fluid the most and a ferromagnetic crystal the least. There is no region of stability for the ferromagnetic fluid, although it comes close at $\Lambda \approx-5$. However, this is a regime where the interaction is strongly attractive, so that perturbation theory is no longer valid, and a trial wave function that includes the possibility of superfluid pairing is required for accurate QMC calculations. It therefore seems unlikely that a weak interaction will have much effect on the phase diagram.

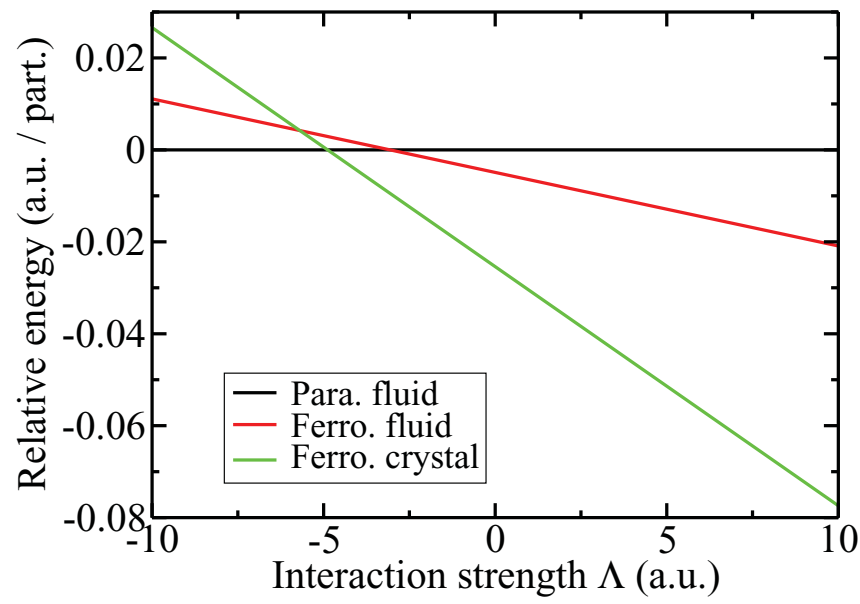

FIG. 18. (Color online) Energy of the ferromagnetic fluid and crystal relative to the energy of the paramagnetic fluid against the strength of the $r^{-3}$ interaction for hard-core particles of diameter $D=0.88$ a.u. 


\section{CONCLUSIONS}

Gases of hard-core particles are a natural model for ultracold-atom systems. Correlation effects in 2D hard-core systems are surprisingly long ranged. Our QMC results show that there is no regime in which itinerant ferromagnetism occurs in a 2D hard-core fluid. As the hard-core diameter $D$ is increased, the system undergoes a transition from a paramagnetic fluid to a crystal at $D=0.83$ a.u. The absence of a region of stability for a ferromagnetic fluid resembles the situation in the 2D HEG. ${ }^{31}$ Including a weak $r^{-3}$ tail in the two-body interaction between hard-core particles does not lead to a significant revision of the phase diagram. We have presented QMC results for the PCFs, static structure factors, and momentum densities of the fluid phases of the hard-core gas.

\section{ACKNOWLEDGMENTS}

We acknowledge financial support from the Leverhulme Trust and the UK Engineering and Physical Sciences Research Council (EPSRC). Computing resources were provided by the Cambridge High Performance Computing Service.
${ }^{1}$ I. Bloch, J. Dalibard, and W. Zwerger, Rev. Mod. Phys. 80, 885 (2008).

${ }^{2}$ S. Giorgini, L. P. Pitaevskii, and S. Stringari, Rev. Mod. Phys. 80, 1215 (2008).

${ }^{3}$ C. Chin, R. Grimm, P. Julienne, and E. Tiesinga, Rev. Mod. Phys. 82, 1225 (2010).

${ }^{4}$ D. J. Papoular, G. V. Shlyapnikov, and J. Dalibard, Phys. Rev. A 81, 041603 (2010).

${ }^{5}$ A. Micheli, G. Pupillo, H. P. Büchler, and P. Zoller, Phys. Rev. A 76, 043604 (2007).

${ }^{6}$ A. V. Gorshkov, P. Rabl, G. Pupillo, A. Micheli, P. Zoller, M. D. Lukin, and H. P. Büchler, Phys. Rev. Lett. 101, 073201 (2008).

${ }^{7}$ N. R. Cooper and G. V. Shlyapnikov, Phys. Rev. Lett. 103, 155302 (2009).

${ }^{8}$ M. Greiner, O. Mandel, T. Esslinger, T. W. Hansch, and I. Bloch, Nature (London) 415, 39 (2002).

${ }^{9}$ R. A. Duine and A. H. MacDonald, Phys. Rev. Lett. 95, 230403 (2005).

${ }^{10}$ G.-B. Jo, Y.-R. Lee, J.-H. Choi, C. A. Christensen, T. H. Kim, J. H. Thywissen, D. E. Pritchard, and W. Ketterle, Science 325, 1521 (2009).

${ }^{11}$ D. Pekker, M. Babadi, R. Sensarma, N. Zinner, L. Pollet, M. W. Zwierlein, and E. Demler, Phys. Rev. Lett. 106, 050402 (2011).

${ }^{12}$ M. Babadi, D. Pekker, R. Sensarma, A. Georges, and E. Demler, e-print arXiv:0908.3483.

${ }^{13}$ C. Nayak, S. H. Simon, A. Stern, M. Freedman, and S. Das Sarma, Rev. Mod. Phys. 80, 1083 (2008).

${ }^{14}$ D. M. Ceperley and B. J. Alder, Phys. Rev. Lett. 45, 566 (1980).

${ }^{15}$ W. M. C. Foulkes, L. Mitas, R. J. Needs, and G. Rajagopal, Rev. Mod. Phys. 73, 33 (2001).

${ }^{16}$ J. B. Anderson, J. Chem. Phys. 65, 4121 (1976).

${ }^{17}$ K. S. Liu, M. H. Kalos, and G. V. Chester, J. Low Temp. Phys. 13, 227 (1973).

${ }^{18}$ C. De Michelis, G. Masserini, and L. Reatto, Phys. Rev. A 18, 296 (1978).

${ }^{19}$ L. Xing, Phys. Rev. B 42, 8426 (1990).

${ }^{20}$ S.-Y. Chang, M. Randeria, and N. Trivedi, Proc. Natl. Acad. Sci. USA 108, 51 (2011).
${ }^{21}$ S. Pilati, G. Bertaina, S. Giorgini, and M. Troyer, Phys. Rev. Lett. 105, 030405 (2010).

${ }^{22}$ R. J. Needs, M. D. Towler, N. D. Drummond, and P. López Ríos, J. Phys. Condens. Matter 22, 023201 (2010).

${ }^{23}$ N. D. Drummond, M. D. Towler, and R. J. Needs, Phys. Rev. B 70, 235119 (2004).

${ }^{24}$ R. J. Needs, M. D. Towler, N. D. Drummond, and P. López Ríos, CASINO version 2.1 User Manual (Cambridge University, Cambridge, UK, 2008).

${ }^{25}$ P. López Ríos, A. Ma, N. D. Drummond, M. D. Towler, and R. J. Needs, Phys. Rev. E 74, 066701 (2006).

${ }^{26}$ C. Lin, F. H. Zong, and D. M. Ceperley, Phys. Rev. E 64, 016702 (2001).

${ }^{27}$ F. H. Zong, C. Lin, and D. M. Ceperley, Phys. Rev. E 66, 036703 (2002).

${ }^{28}$ N. D. Drummond, Z. Radnai, J. R. Trail, M. D. Towler, and R. J. Needs, Phys. Rev. B 69, 085116 (2004).

${ }^{29}$ B. Tanatar and D. M. Ceperley, Phys. Rev. B 39, 5005 (1989).

${ }^{30}$ F. Rapisarda and G. Senatore, Aust. J. Phys. 49, 161 (1996).

${ }^{31}$ N. D. Drummond and R. J. Needs, Phys. Rev. Lett. 102, 126402 (2009).

${ }^{32}$ M. Li, H. Fu, Y.-Z. Wang, J. Chen, L. Chen, and C. Chen, Phys. Rev. A 66, 015601 (2002).

${ }^{33}$ T. Kato, Commun. Pure Appl. Math. 10, 151 (1957); R. T. Pack and W. B. Brown, J. Chem. Phys. 45, 556 (1966).

${ }^{34}$ C. J. Umrigar, M. P. Nightingale, and K. J. Runge, J. Chem. Phys. 99, 2865 (1993).

${ }^{35}$ N. D. Drummond and R. J. Needs, Phys. Rev. B 79, 085414 (2009).

${ }^{36}$ S. Chiesa, D. M. Ceperley, R. M. Martin, and M. Holzmann, Phys. Rev. Lett. 97, 076404 (2006).

${ }^{37}$ N. D. Drummond, R. J. Needs, A. Sorouri, and W. M. C. Foulkes, Phys. Rev. B 78, 125106 (2008).

${ }^{38}$ H. Akima, J. Assoc. Comput. Math. 17, 589 (1970).

${ }^{39}$ The energy differences between different spin configurations in antiferromagnetic crystals are much too small for us to resolve. The particular antiferromagnetic spin configuration that we have considered has alternating stripes of up- and down-spin particles. Each particle has two same-spin neighbors and four opposite-spin neighbors. 